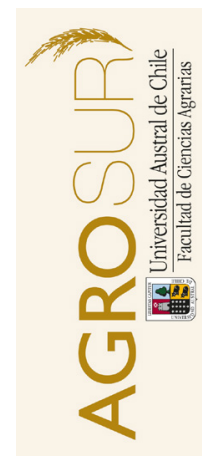

\title{
Diferentes tipos de pre-tratamiento de la pulpa del café para generar su aprovechamiento
}

\section{Different types of pre-treatment of coffee pulp to promote its use}

\author{
Castillo, A. ${ }^{a, b *}$, Muñoz, A. ${ }^{a}$, Pantoja, R..$^{a, e}$, Portilla, M..$^{a, c, d}$ \\ ${ }^{a}$ Programa de Ingeniería Ambiental, Facultad de Ingeniería, Universidad Mariana, Pasto, Colombia. \\ ${ }^{b}$ Programa de Maestría en Ciencias Agropecuarias, Facultad de Agronomía y Veterinaria, \\ Universidad Autónoma de San Luis Potosí, México. \\ ${ }^{c}$ Grupo de Investigación Ambiental GIA - Categoría B COLCIENCIAS, Pasto, Colombia \\ ${ }^{d}$ Escuela de Postgrados, Facultad de Ciencias Ambientales Universidad Tecnológica de Pereira, Pereira, Colombia. \\ ${ }^{e}$ Programa Ingeniería Agroindustrial, Universidad de Nariño, Nariño, Colombia.
}

\begin{tabular}{l} 
A R T I C L E I N F O \\
\hline Article history: \\
Received 15.09.2018 \\
Accepted 12.12.2018 \\
\hline Keywords: \\
Pre-treatment \\
Pleurotus ostreatus \\
Alcoholic fermentation \\
Reducing sugars \\
\hline Original Research Article \\
Food Science \\
\hline *Corresponding author: \\
Anyela Nayive Castillo Solarte \\
E-mail address: \\
anycastillo@umariana.edu.co
\end{tabular}

A B S T R A C T

The present investigation evaluated the process of delignification of the coffee pulp as a residue of the agroindustry through the alcoholic fermentation process that results from a biological pre-treatment, using the white rot fungus Pleurotus ostreatus L. which is recognized by its easy adaptation to the medium and hydrolytic capacity on lignin, and also for having a high nutritional potential for human consumption. Four different contact times between the substrate and the fungus (40, 30, 25, 20 days) were assesed. The average percentage of delignification in each analyzed time presented statistically significant differences $(\mathrm{p}<0.05)$. However, a greater percentage of delignification $(51.422 \%)$ was registered in the sample evaluated at 30 days, allowing to improve the utilization of the by-product when compared with the chemical pre-treatment carried out using an acidic and alkaline medium, where the percentage of delignification achieved is $42.5144 \%$ and $40.713 \%$, respectively. The alcoholic fermentation process was carried out using the Saccharomyces cerevisiae Meyen ex E.C.Hansen yeast on the different delignified substrates with the different pre-treatments (biological 30 days, acid repeat 1 , alkaline repetition 3 and without hydrolysis). To recognize the efficiency of this process, the concentration of reducing sugars at the beginning and at the end of the fermentation were quantified. There was a higher availability in the biological pre-treatment sample $\left(89.51 \mathrm{~g} \mathrm{~L}^{-1}\right)$ and with a higher percentage of consumption of the sugars during the process when compared with the other samples evaluated.

\section{RESUMEN}

Se evaluó el proceso de deslignificación de la pulpa de café como residuo de su agroindustria, a través de un proceso de fermentación alcohólica, mediante un pre-tratamiento biológico, usando el hongo Pleurotus ostreatus L. de la pudrición blanca, basidiomiceto reconocido por su fácil adaptación al medio y su capacidad hidrolítica sobre la lignina, además de contar con un alto potencial nutritivo para el consumo humano, este procedimiento se realizó durante 4 tiempos diferentes de contacto entre el sustrato y el hongo (40, 30, 25, 20 días). El promedio del porcentaje de deslignificación en cada tiempo analizado presenta diferencias estadísticamente significativas $(\mathrm{p}<0,05)$, sin embargo, se registró mayor porcentaje de deslignificación $(51,422 \%)$ en la muestra evaluada a 30 días, además de permitir mejorar el aprovechamiento del subproducto, en comparación con el pre-tratamiento químico que se llevó a cabo a partir de la utilización de un medio ácido y alcalino donde el porcentaje de deslignificación alcanzado fue de 42,5144\% y 40,713\%, respectivamente. Continuamente el proceso de fermentación alcohólica se llevó a cabo mediante la utilización de la levadura Saccharomyces cerevisiae Meyen ex E.C.Hansen sobre los diferente sustratos deslignificados mediantelos diferentes pre-tratamientos (biológico 30 días, ácido repetición 1, alcalino repetición 3 y sin hidrólisis), finalmente, para reconocer la eficiencia de este proceso se cuantificó concentración de azúcares reductores al inicio y al final de la fermentación, presentando mayor disponibilidad de los mismos en la muestra del pre-tratamiento biológico $\left(89,51 \mathrm{~g} \mathrm{~L}^{-1}\right)$ y con mayor porcentaje de consumo de los azúcares durante el proceso, en comparación con las otras muestras evaluadas.

Palabras clave: Pre-tratamiento, Pleurotus ostreatus, fermentación alcohólica, azúcares reductores.

\section{INTRODUCCIÓN}

En los últimos años el crecimiento en la producción de café en Colombia se ha incrementado significativa- mente, según lo establece la Federación Nacional de Cafeteros de Colombia (2013). Esta situación ha venido generando beneficios sobre la economía del país, y en general, en las regiones donde se realiza esta actividad 
productiva, se debe a esto, que los recientes debates relacionados con el desarrollo sostenible en comunidades rurales atañen de manera directa a la caficultura según Balseca y Cabrera (2011). En este contexto el Municipio de Buesaco Nariño se ve altamente influenciado por esta labor.

Sin embargo, el beneficio de la agroindustria del café trae consigo un aumento en la generación de residuos agroindustriales según Rodríguez y Jaramillo, (2004) plantean que al final del ciclo productivo, los subproductos se convierten en un residuo de tipo contaminante si no se efectúa una debida manipulación, debido a que pierden y adquieren ciertas características que los hacen desfavorables dentro del ecosistema, acrecentado las emisiones de gases efecto invernadero, contaminación del suelo y cuerpos de agua la región, los cuales varían de acuerdo al proceso, manejo y aprovechamiento que se da sobre los mismos.

En Colombia solamente se aprovecha el 5\% del peso del fruto fresco en la preparación de la bebida, el 95\% restante está representado en residuos lignocelulósicos como mesocarpio, mucilago, tallos, entre otros Rodríguez y Zambrano (2010). De esta forma el actual aprovechamiento y disposición que se está proporcionando sobre el alto volumen del material residual del proceso está generando impactos negativos en la calidad de los cuerpos de agua y suelos fértiles; lo que representa la existencia de una agricultura insostenible

Rodríguez y Zambrano (2010) afirman que el promedio de la producción de pulpa en Colombia es de $2,25 \mathrm{t} \mathrm{ha}^{-1} \mathrm{año}^{-1}$, y por cada millón de sacos de $60 \mathrm{~kg}$ de café almendra que Colombia exporta se generan $162.900 \mathrm{t}$ de pulpa fresca que si no se utiliza adecuadamente producirán una contaminación equivalente a la generada durante un año de excretas y orinas de una población de 868.736 habitantes.

En esta medida, se debe destacar que actualmente en la mayoría de las fincas cafeteras del municipio de Buesaco Nariño se realiza dicho proceso sin un aprovechamiento total de la materia prima (Alcaldía Municipal de Buesaco, 2008). No obstante, el desarrollo de tecnologías ambientales ha permitido reconocer que estos residuos tienen un alto potencial beneficiario al ser tratados y aprovechados de forma diferente Rodríguez (2007).

Bajo esta situación en el desarrollo de la presente investigación se implementó un sistema biotecnológico de fácil adaptación al contexto poblacional, evaluado estratégicamente dentro del marco de lo efectivo. Esto se consiguió a partir del pre-tratamiento de deslignificación biológica de la pulpa de café, mediante el cultivo del hongo Pleurotus ostreatus L., y del análisis comparativo a partir de un proceso de fermentación alcohólica, contribuyendo finalmente a solucionar el impacto de los residuos del café en el ambiente y constituyendo una alternativa de negocio para los productores de café (Gonzáles y Molina, 2009).
De esta forma es evidente reconocer la oportunidad que representa el pre-tratamiento al facilitar los mecanismos de generación de bioetanol para el caficultor, en esta medida Marques et al. (1999) mencionan que resulta de gran interés evaluar la biotecnología a partir del uso de Pleurotus, como una alternativa sostenible por su capacidad hidrolítica, permitiendo la despolimerización de estos materiales lignocelulósicos en azúcares fermentables, a esto se suma la actividad comercial que se puede generar a partir del hongo cultivado en el proceso de deslignificación, generando así posibles fuentes de ingresos económicos para la comunidad caficultora debido a que este es un producto con alta cantidad de proteínas que aportan gran parte de los aminoácidos esenciales para la dieta de las personas, además de su valor medicinal, convirtiéndose así en un proceso productivo de mayor rentabilidad (Cardona y Bedoya, 1996).

\section{MATERIALES Y MÉTODOS}

La investigación se desarrolló en el Municipio de Buesaco Nariño, en la finca El Guayabo y en el laboratorio de física y química de los laboratorios de la Universidad Mariana- sede Monte Alvernia.

Los materiales requeridos para el desarrollo de la investigación fueron: Pulpa de café, la cual se obtuvo del proceso de despulpado de café cereza Variedad Castillo ${ }^{\circledR}$, sin seleccionar; además se utilizó el hongo, Pleurotus ostreatus para el pre-tratamiento biológico productor de la enzima celulasa, por otra parte en el proceso de fermentación alcohólica se evaluó la cepa comercial Saccharomyces cervisiae seca.

Los tratamientos consistieron en valuar 2 pre-tratamientos, así: uno mediante hidrólisis biológica $(40,30$, 25 , y 20 días) y uno a partir de medios hidrolizantes químicos (ácido y alcalino); la unidad experimental estuvo conformada por una muestra de pulpa de $0,5 \mathrm{~kg}$.

Para el pre-tratamiento biológico se planteó un diseño experimental unifactorial, considerando como única variable el tiempo de retención del hongo en el sustrato, con cuatro niveles, donde la variable de respuesta es el porcentaje de lignina removida. Asimismo para el pre-tratamiento químico se presenta un diseño unifactorial donde la variable es el medio hidrolizante con dos niveles.

En cuanto al proceso de fermentación se realizó un diseño experimental unifactorial con una variable independiente correspondiente al tipo de pre-tratamiento con cuatro niveles, sobre la variable respuesta concentración de azúcares reductores (Figura 1).

En materia del desarrollo procedimental se tuvo en cuenta las siguientes condiciones:

- Se utilizó pulpa fresca, con menos de $1 \mathrm{~h}$ de generada. 
- Pre-tratamiento biológico.

Variable Independiente
\begin{tabular}{|l|}
\hline Tiempo \\
Niveles: \\
40, 30, 25, 20 días
\end{tabular}

\section{Grupo}

Repeticiones: 9

Total: 36 muestras de

$500 \mathrm{~g}$ de pulpa de café
Variable dependiente

Porcentaje de lignina

removida.

Factores de Confusión: Temperatura, humedad relativa, rendimiento del cultivo.

- Pre-tratamiento químico.

\begin{tabular}{l|l|l|}
\multicolumn{1}{|l|}{ Variable Independiente } & Grupo \\
\begin{tabular}{|l|l|l|}
\hline Medio hidrolizante & Repeticiones: 3 \\
Total: 6 muestras de & Porcentaje de lignina \\
N00g de pulpa de café
\end{tabular} & \begin{tabular}{l} 
removida. \\
\hline
\end{tabular}
\end{tabular}

Factores de Confusión: Tiempo

- Fermentación alcohólica

Variable Independiente

Pre-tratamiento.

Niveles: Biológico,

Acido, Alcalino, Sin

Hidrólisis.

\section{Grupo}

Total: 4 muestras de mosto de pulpa de café
Variable dependiente

Concentración de azúcares reductores

Factores de Confusión: Temperatura, Tiempo, pH.

Figura 1. Diseño experimental.

Figure 1. Experimental design.

- La hidrólisis ácida se realizó con ácido sulfúrico concentrado $\left(17,4 \mathrm{~mL} \mathrm{~kg} \mathrm{de} \mathrm{pulpa}^{-1}\right)$ y se adicionó $\mathrm{Na}_{2} \mathrm{SO}_{3}\left(22,5 \mathrm{~g} \mathrm{~kg}\right.$ de pulpa $\left.^{-1}\right)$. Luego, el material se sometió a baño agua maría durante 20 min.

- La hidrólisis alcalina se realizó con hidróxido de sodio al 32\% (20 mL kg de pulpa-1 y se adicionó $\mathrm{Na}_{2} \mathrm{SO}_{3}$ $\left(22,5 \mathrm{~g} \mathrm{~kg} \mathrm{de} \mathrm{pulpa}^{-1}\right)$. Posteriormente, el material se sometió a baño agua maría durante $20 \mathrm{~min}$.

- El pre-tratamiento biológico se llevó a cabo en utilización del hongo Pleurotus ostreatus, se empleó una taza de inoculación del 15\%, el material se sometió previamente a un proceso de selección y adecuación y a partir del uso de un invernadero se proporcionó un microclima con la finalidad de llevar el registro de algunas de las variables ambientales (temperatura y humedad relativa)

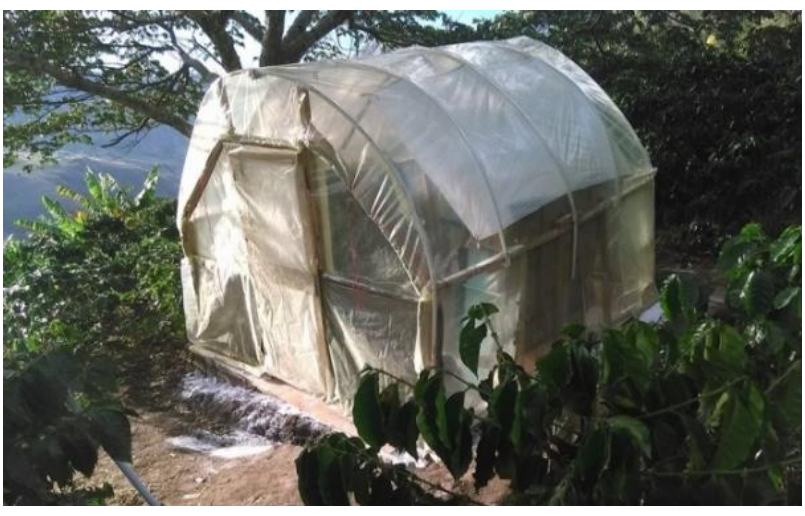

Figura 2. Invernadero.

Figure 2. Greenhouse. según lo requería cada etapa de crecimiento del hongo (Figura 2). 
A continuación en la Figura 3 se indica un registro fotográfico de cada una de las etapas del crecimiento del hongo sobre el sustrato, en el proceso de deslignificación biológica.

Una vez terminada la etapa de hidrólisis, se determinó la concentración de azúcares reductores y el pH del mosto obtenido se adecuó a un valor de 5,0 \pm 0,5 utilizando ácido sulfúrico o hidróxido de sodio, y se adicionó la cepa de levadura en una proporción de $2,5 \% \mathrm{p} / \mathrm{v}$, a una temperatura de incubación de $30{ }^{\circ} \mathrm{C}$, y después de 24 horas de fermentación se cuantificó nuevamente la concentración de azúcares reductores.

- Para el proceso de fermentación alcohólica se utilizó una relación de 1,5 L de agua por 1,0 kg de pulpa fresca.

Finalmente los datos fueron sometidos a un análisis estadístico por cada diseño experimental (deslignificación y fermentación alcohólica) mediante el programa SPCS.

\section{RESULTADOS Y DISCUSIÓN}

Los hongos de la producción blanca son reconocidos por presentar fácil adaptación con el medio, sin embargo, para garantizar que sobre la variable de respuesta (porcentaje de remoción de lignina) (Chávez-Sinfonte y Domine, 2006) no influya ningún otro factor se buscó proporcionar condiciones de temperatura, humedad e intensidad lumínica constantes y apropiadas para cada etapa del crecimiento del hongo. En este sentido, se obtuvo un registro del control de temperatura y humedad relativa realizados durante los 40 días, y un análisis estadístico descriptivo de los mismo comprobando que el invernadero y el sistema de seguimiento en general, si proporcionaron un control de las condiciones requeridas para el desarrollo del pre-tratamiento.

En el Cuadro 1 se puede observar los valores máximos y mínimos registrados en el control diario, tanto para la temperatura como para la humedad, reconociéndose que se encuentran dentro de los rangos óptimos de crecimiento del hongo establecidos por Fernández et al. (2015).

Después de llevar a cabo el proceso pertinente a la degradación de lignina por acción enzimática del hongo en todas las fases del cultivo, se identificó que no existieron pérdidas o muestras improductivas con respecto al número total de montículos inoculados.

Los resultados de los componentes de rendimiento, como eficiencia biológica y variables morfológicas en las etapas productivas, que garantizan que el tratamiento biológico se haya llevado a un buen término Núñez (2015), se indican a continuación:

\section{Eficiencia biológica}

Los resultados de eficiencia obtenidos son producto de diferentes tiempos de retención del hongo en el sus-

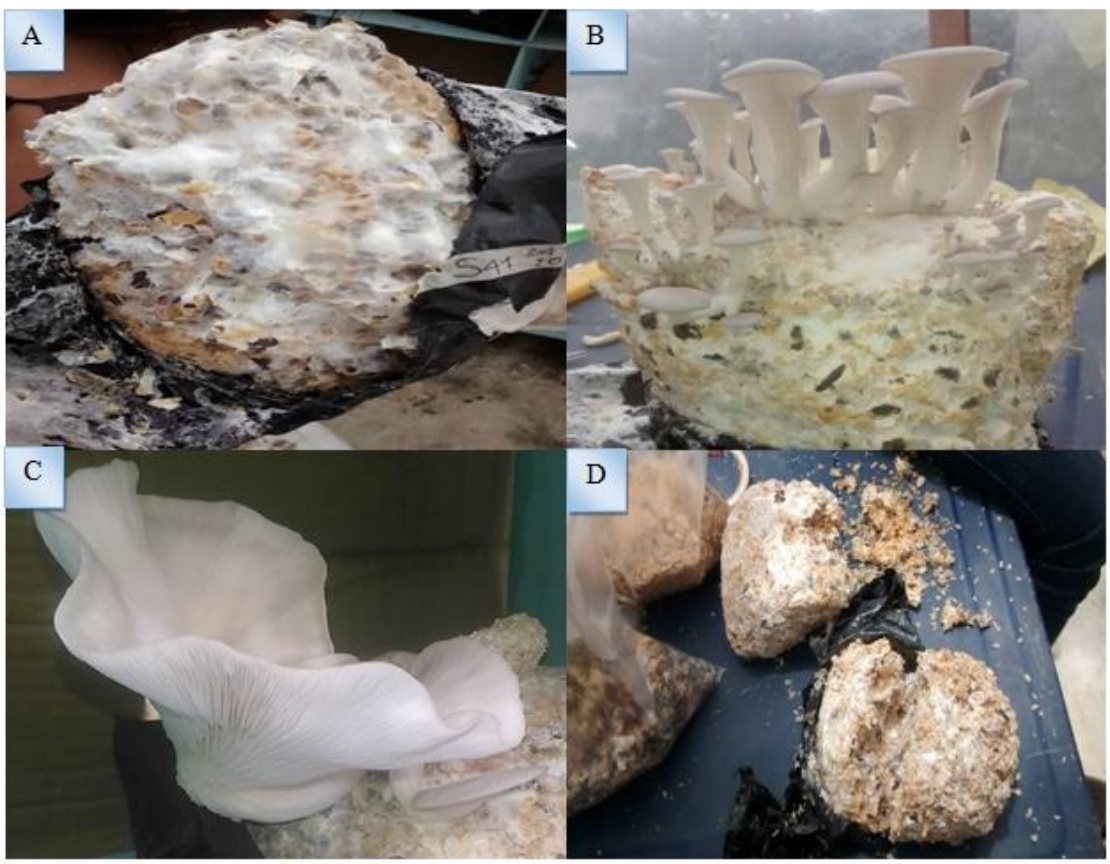

Figura 3. A. Proceso del cultivo; B. Inoculación del micelio; C. Fructificación; D. Retiro del sustrato.

Figure 3. A. Cultivation process; B. Inoculation of the mycelium; C. Fructification; D. Removal of the substrate. 
trato (40, 30, 25 y 20 días), lo que representó que las muestras sembradas en los dos primeros tiempos tuvieran un 74,5\% de la producción total, puesto que en éstas se alcanzaron a realizar dos cosechas, siendo la primera más fructífera, en lo que respecta a los siguientes dos tiempos de siembra, se presentó una reducción de esta variable al 25,5\% aproximadamente, por razón de lo expresado anteriormente.

\section{Variables morfológicas}

Los resultados de estas variables consistieron en la determinación del número de fructificaciones por bolsas y medidas biométricas (longitud del sombrero y longitud de estípite de los carpóforos), en el Cuadro 2 se presentan los valores medios para cada parámetro de análisis anteriormente expuesto.

\section{Cuantificación del porcentaje de deslignificación}

Los resultados se fundamentaron en identificar el porcentaje de remoción de lignina en cada una de las muestras de los diferentes tratamientos (biológico y químico), los cuales se obtuvieron mediante la aplicación de la metodología número Kappa, propuesta por Salcedo y Pardo (2012) y el análisis Van Soest propuesto por García Criado et al. (1977).

Los resultados obtenidos fueron sometidos a análisis estadísticos, de esta forma con respecto al tratamiento biológico mediante la metodología número Kappa, reportó que el promedio del porcentaje de deslignificación no es igual para cada tratamiento evaluado en los diferentes días (Cuadro 3), lo cual representa que la variable tiempo de contacto del hongo con el sustrato, si presenta diferencias en los resultados del proceso.

Cuadro 1. Valores máximos y mínimos registrados de temperatura y humedad.

Table 1. Maximum and minimum registered values of temperature and humidity.

\begin{tabular}{lccccc}
\hline & N & Mínimo & Máximo & Media & Desviación estándar \\
\hline Temperatura & 286 & 17,00 & 28,00 & 23,55 & 2,45 \\
Humedad & 286 & 55,00 & 80,00 & 66,74 & 3,39 \\
N válido & 286 & & & & \\
\hline
\end{tabular}

Cuadro 2. Medias de las variables morfológicas.

Table 2. Means of morphological variables.

\begin{tabular}{cccc}
\hline \multirow{2}{*}{ Tiempo (días) } & \multirow{2}{*}{$\begin{array}{c}\text { Media del } \mathbf{N}^{\circ} \text { de } \\
\text { fructificaciones por bolsas }\end{array}$} & \multicolumn{2}{c}{ Medidas biométricas } \\
\cline { 3 - 4 } & 46,40 & 2,20 & 5,30 \\
\hline 40 & 43,20 & 2,20 & 4,70 \\
25 & 17,70 & 2,10 & 5,40 \\
20 & 14,90 & 2,20 & 5,30 \\
\hline
\end{tabular}

Cuadro 3. Prueba ANOVA del porcentaje de deslignificación del tratamiento biológico.

Table 3. ANOVA test of percentage of de-lignification of the biological treatment.

\begin{tabular}{lccccc}
\hline & Suma de cuadrados & gl & Media cuadrática & F & Sig. \\
\hline Entre grupos & 665,26 & 3 & 221,75 & 13,57 & 0,00 \\
Dentro de grupos & 130,68 & 8 & 16,33 & & \\
\hline Total & 795,99 & 11 & & \\
\hline
\end{tabular}

Ho: El promedio de porcentaje de deslignificación es igual para cada tratamiento

Ha: El promedio de porcentaje de deslignificación no es igual para cada tratamiento 
Asimismo, mediante un análisis Post Hoc de diferencias de medias significativas de los resultados del tratamiento biológico, se reconoció que en promedio el porcentaje de deslignificación de los tratamiento realizados durante los días 20 y 25 son iguales, al igual que entre los tratamiento con 40 y 30 días de contacto entre el hongo y el sustrato, sin embargo, se selecciona el tratamiento llevado a cabo a 30 días debido a que en los resultados reportados individualmente se presenta mayor eficiencia en la remoción de lignina.

En cuanto al tratamiento químico, la prueba ANOVA reportó que en promedio cada tratamiento (alcalino y ácido) tiene igual porcentaje de deslignificación (Cuadro 4), no obstante, en los resultados individuales si se identifica una variación de los mismos.
Para la selección del resultado más representativo del pre-tratamiento químico se tomó como punto de referencia un proceso estadístico mediante cartas de control, además del concepto teórico que define la metodología de número Kappa (Figura 4 y 5).

De esta forma los resultados seleccionados por cada tratamiento se resumen en el Cuadro 5, donde se puede observar la diferencia del porcentaje de deslignificación por cada uno, y reconocer que según la metodología de número Kappa el pre-tratamiento biológico presenta mayor remoción, seguida del pre-tratamiento ácido y alcalino.

En esta medida resultó pertinente llevar a cabo la segunda prueba de laboratorio, la cual representa mayor precisión en los resultados, por lo tanto, las mues-

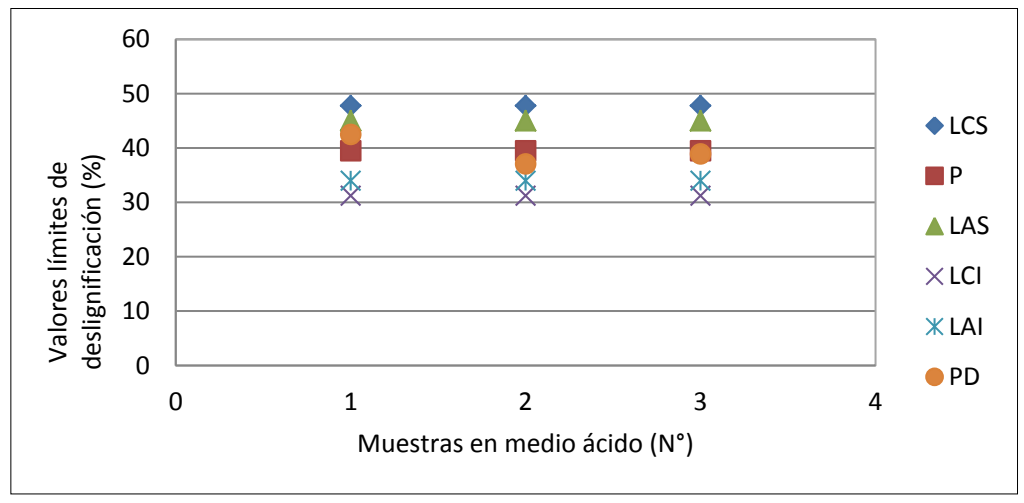

Figura 4. Gráfica de control pre-tratamiento químico medio ácido. P: promedio, LAS: límite de alarma superior, LCS: límite de control superior, LAI: límite de alarma inferior, LCI: límite de control inferior, PD: porcentaje de remoción.

Figure 4. Acid chemical pre-treatment control chart. P: Mean, LAS: Upper alarm limit, LCS: Upper control limit, LAI: Lower alarm limit, LCI: Lower control limit, PD: Removal percentage.

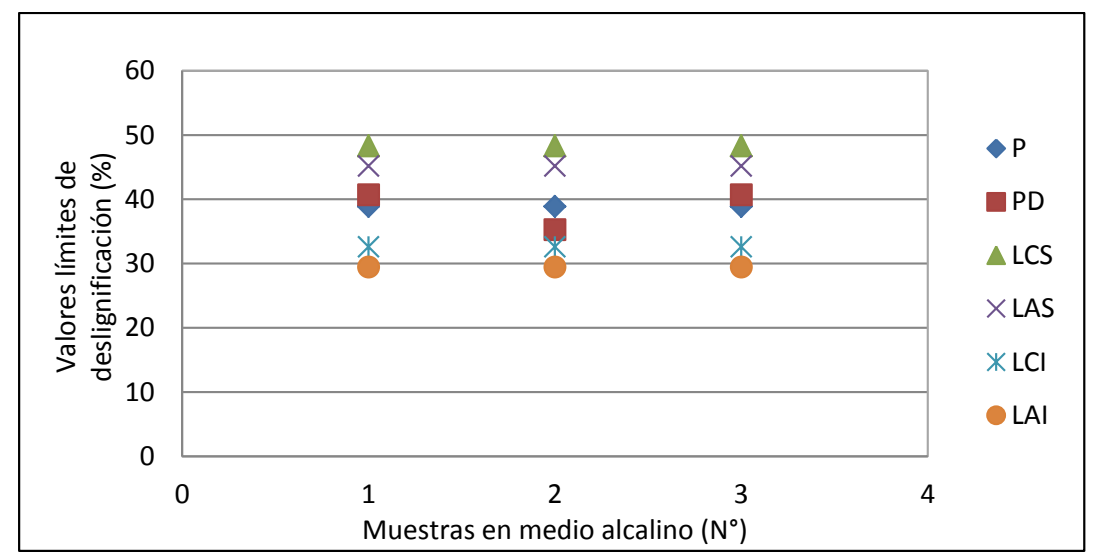

Figura 5. Gráfica de control pre-tratamiento químico medio alcalino. P: Promedio, LAS: Limite de alarma superior, LCS: Limite de control superior, LAI: Limite de alarma inferior, LCI: Limite de control inferior, PD: Porcentaje de remoción.

Figure 5. Alkaline chemical pre-treatment control chart. P: Mean, LAS: Ipper alarm limit, LCS: Upper control limit, LAI: Lower alarm limit, LCI: Lower control limit, PD: Removal percentage. 
tras pre-seleccionadas en el proceso anterior se sometieron a análisis Van Soest, que en consideración de una muestra inicial sin pre-tratamiento, y por diferencia de pesos, se obtuvieron los resultados del contenido de lignina presente en la muestra y se logró reconocer el porcentaje de deslignificación.

A continuación se presenta en el Cuadro 6 los resultados del análisis en base húmeda y seca, con el correspondiente dato de porcentaje para cada tratamiento.

El contenido de lignina se calculó teniendo en cuenta la pérdida de peso original de la fibra, obtenida por el método Fibra Detergente Ácido (FDA), en esta medida se consideró el peso de los sustratos antes y después de cada tratamiento aplicado. El residuo obtenido determina el complejo celulósico, mediante la digestión

Cuadro 4. Prueba ANOVA del porcentaje de deslignificación del tratamiento químico.

Table 4. ANOVA test of percentage of delignification of the chemical treatment.

\begin{tabular}{lccccc}
\hline & Suma de cuadrados & gl & Media cuadrática & F & Sig. \\
\hline Entre grupos & 0,55 & 1 & 0,55 & 0,06 & 0,82 \\
Dentro de grupos & 34,97 & 4 & 8,74 & & \\
\hline Total & 35,52 & 5 & & \\
\hline
\end{tabular}

Ho: El promedio de porcentaje de deslignificación es igual para cada tratamiento

Ha: El promedio de porcentaje de deslignificación no es igual para cada tratamiento

Cuadro 5. Porcentaje de deslignificación Numero Kappa.

Table 5. Percentage of de-lignification Kappa number.

\begin{tabular}{lccc}
\hline Pre-tratamiento & Descripción & $\mathrm{k}$ & \% de deslignificación \\
\hline Biológico & 30 días & 194,78 & 51,42 \\
Ácido & Repetición numero 1 & 230,49 & 42,51 \\
Alcalino & Repetición numero 3 & 237,71 & 40,71 \\
\hline
\end{tabular}

Cuadro 6. Resumen de resultados análisis Van Soest.

Table 6. Summary of results Van Soest analysis.

\begin{tabular}{|c|c|c|c|}
\hline \multicolumn{4}{|c|}{ Van Soest secuencial, bolsas Ankom } \\
\hline Tratamiento & Base húmeda (g $100 \mathrm{~g}^{-1}$ ) & Base seca (g $\left.100 \mathrm{~g}^{-1}\right)$ & \% de deslignificación \\
\hline Biológico & 1,80 & 20,60 & 36,22 \\
\hline Ácido & 3,82 & 26,40 & 18,26 \\
\hline Alcalino & 3,83 & 25,60 & 20,74 \\
\hline Sin pre-tratamiento & 2,67 & 32,30 & 0,00 \\
\hline \multicolumn{4}{|c|}{ Van Soest secuencial, oxidación $\mathrm{KMnO}_{4}$} \\
\hline Tratamiento & Base húmeda (g $100 \mathrm{~g}^{-1}$ ) & Base seca (g $100 \mathrm{~g}^{-1}$ ) & \% de deslignificación \\
\hline Biológico & 5,40 & 8,60 & 57,84 \\
\hline Ácido & 1,74 & 12,00 & 41,17 \\
\hline Alcalino & 1,74 & 11,60 & 43,14 \\
\hline Sin pre-tratamiento & 0,30 & 20,40 & 0,00 \\
\hline
\end{tabular}


de la muestra seca, con un detergente en un amortiguar ácido. La FDA se empleó como paso preliminar para la determinación de lignina, y puede correlacionarse con la digestibilidad de un material fibroso.

\section{Fermentación}

Teniendo en cuenta que, para llevar a cabo este proceso se requiere que en el medio propiciado por microorganismos, interactúen parámetros específicos y condiciones especiales, se estimaron los valores de $\mathrm{pH}$ al final del proceso de fermentación. El pH óptimo para las levaduras se encuentra en un rango entre 4,5 y 6,0 (Ospina y Palacios, 1994), de esta manera únicamente el tratamiento alcalino presentó valores por encima de 5,0 , con respecto al tratamiento biológico con el que se alcanzó el mayor rendimiento en concentración de azúcares reductores, presentó valores de pH entre 4,3 y 5,0.

Por otra parte, los ${ }^{\circ}$ Brix que determinan el contenido de sólidos solubles presentes en el jugo fermentado de pulpa, donde se encuentran azúcares, ácidos, sales y demás compuestos solubles en agua, permitieron observar en el presente estudio, que el tratamiento con mayor porcentaje de ${ }^{\circ}$ Brix en el mosto fermentado (pre-tratamiento biológico) fue el que presentó mayor consumo de azúcares fermentables y menores valores de pH (Gonzáles y Molina, 2009).

En relación a los parámetros densidad y ${ }^{\circ}$ Brix, se obtuvo que al disminuir la densidad en el tiempo reduce los ${ }^{\circ}$ Brix, esto se debe a que en el mosto se presenta un consumo de los azúcares, y por lo tanto, su masa ocupa un menor volumen. Asimismo representa de forma indirecta la formación de bioetanol. De igual manera, transcurridas las 18 horas la densidad toma un comportamiento estable, indicando la estabilización de la concentración de azúcares.

\section{Concentración de azúcares reductores}

La obtención de los resultados de concentración de azúcares reductores en las muestras pre-tratadas se identificó mediante una curva patrón de glucosa. Em- pleando la ecuación resultante, y con los resultados de absorbancia de las muestras de los cuatro procesos de evaluación (biológico, ácido, alcalino y sin hidrólisis) se calculó la concentración de azúcares reductores, obteniendo los resultados expuestos en el Cuadro 7.

La razón por la que se registró mayor concentración de azúcares simples en el mosto preparado con el sustrato deslignificado a partir del hongo antes de fermentar, y que presentó mayor consumo de la concentración de azúcares con una diferencia de 17,5 g L $\mathrm{g}^{-1}$ después de la fermentación, se atribuye a que la muestra tratada mediante el proceso biológico presentaba menor cantidad de lignina con respecto a las otras muestras (Bonifacino, 2012).

Debido a que en el proceso de deslignificación se requiere someter al sustrato en contacto con medios hidrolizantes, resultó que el utilizar hongos de la pudrición blanca en un determinado tiempo, favorece el porcentaje de deslignificación. De esta manera, en la investigación llevada a cabo por Rodríguez y Jaramillo, (2004) se reporta el tiempo de actuación del hongo efectivo sobre los sustratos de café, el cual es de 30 a 45 días. En contraste con la presente investigación se indica que, el porcentaje con mayor remoción de lignina se encuentra en las pruebas con mayor tiempo de contacto.

Asimismo, es posible analizar las causas que generaron la variación entre los resultados de los pretratamientos más comúnmente usados en procesos de deslignificación. En esta medida se debe reconocer que como lo cita Juri Awad (2011), los hongos de la pudrición blanca presentan alta eficiencia en el proceso de deslignificación, esto en consecuencia, de su actividad metabólica permitiendo solubilizar y digestibilizar la lignina presente en la pulpa.

En cuanto a los resultados obtenidos por el tratamiento ácido, que para efectos prácticos es el tratamiento que representó menor porcentaje de remoción de la lignina en la muestra, se puede inferir que esto se generó en consecuencia de que el ácido reacciona disolviendo complejo hemicelulolítico, pero que disminuye su capacidad de disolución al entrar en contacto con los polímeros que conforman la lignina (Ballesteros et al., 2006).

Cuadro 7. Concentración de azúcares reductores.

Table 7. Concentration of reducing sugars.

\begin{tabular}{lcccc}
\hline \multirow{2}{*}{ Muestra } & \multicolumn{2}{c}{ Inicial } & \multicolumn{2}{c}{ Final } \\
\cline { 2 - 5 } & Absorbancia & Concentración & Absorbancia & Concentración \\
\hline P.S.H & 0,24 & 35,60 & 0,36 & 33,91 \\
P. Biológico & 0,52 & 89,51 & 0,60 & 72,46 \\
P. Alcalino & 0,35 & 69,83 & 0,38 & 62,82 \\
P. Ácido & 0,45 & 53,95 & 0,42 & 48,44 \\
\hline
\end{tabular}


Por otra parte, para asegurar un resultado favorable durante el proceso de fermentación, se requiere que existan condiciones especiales y parámetros específicos. De este modo, resulta pertinente resaltar que el $\mathrm{pH}$ óptimo para las levaduras se encuentra en un rango entre 4,5 y 6,0 Ospina y Palacios, (1994). Sin embargo, estudios realizados por Fajardo y Sarmiento (2007) reportan que el pH más favorable para el crecimiento de $S$. cerevisiae se encuentra en el rango entre 4,4 y 5,0, con un $\mathrm{pH}$ de 4,5 para su crecimiento óptimo, pero no se desarrolla bien en medio alcalino, a menos que se haya adaptado previamente al mismo. El pH del mosto tiene influencia sobre los productos finales obtenidos en el proceso de fermentación anaerobia, y tiende a disminuir debido a la producción de ácidos.

En el proceso de generación de etanol se alcanzó para la muestra tratada con el método biológico valores de densidad alrededor de $1,2 \mathrm{~g} \mathrm{ml}^{-1}$, resultado que según Sánchez y Cardona (2005) indica que la densidad al bajar a su punto máximo (1100 $\left.\mathrm{g} \mathrm{L}^{-1}\right)$ hasta estabilizarse, presenta una fermentación exitosa.

Finalmente, se observa la relación que existe entre la cantidad de lignina en las muestras, y la disponibilidad y consumo de azúcares reductores en el proceso de fermentación; cabe señalar que el tratamiento que permite mejorar la fermentación de la pulpa de café después del método biológico es el tratamiento alcalino, permitiendo un consumo de 7,01 $\mathrm{g} \mathrm{L}^{-1}$ de azúcares reductores.

Teniendo en cuenta los resultados de concentración de azúcares simples para el proceso de fermentación después de cada tratamiento, se puede afirmar que la mayor disponibilidad de los mismos presentes en el mosto, representa mayor generación de bioetanol. En el estudio de generación de bioetanol mediante la fermentación alcohólica de la pulpa de café realizado por Rodríguez (2007), se reportan resultados de la evaluación de diferentes tratamientos, indicando que, los mayores promedios de los contenidos de etanol se alcanzaron para los tratamientos donde se utilizó la enzima celulasa, que corresponde a la enzima secretada por el hongo valorado en la presente investigación, con un $97,78 \%$ de etanol, y una concentración inicial de azúcares reductores equivalente a $93,29 \mathrm{mg} \mathrm{ml}^{-1}$.

Es importante tomar en cuenta que la concentración de azúcares reductores reconocida en la presente investigación, indica que la pulpa de café es un subproducto agrícola promisorio a ser utilizado en la obtención de alcoholes, donde su uso no sólo se puede limitar a la generación de bebidas alcohólicas, sino también, que permiten apostar al uso de éste como una fuente de recursos energéticos (Davis et al., 2010).

\section{CONCLUSIONES}

El análisis del contenido de lignina, de cada muestra antes y después del tratamiento aplicado, indico como resultado que el pre-tratamiento biológico, alcanza mayor porcentaje de remoción, pues, los hongos de la pudrición blanca gracias a su actividad enzimática sobre la pulpa de café actúan ejerciendo una alta despolimerización de la lignina, favoreciendo el proceso fermentación alcohólica.

En el pre-tratamiento biológico, se reconocieron diferencias significativas en el grado de deslignificación, en función del tiempo de cosecha. De esta forma, el pre-tratamiento realizado a 30 días presentó mayor eficiencia en el proceso de deslignificación $(57,84 \%)$ con respecto a los demás.

Los subproductos de la agroindustria del café, pueden adquirir un valor económico agregado si se desarrollan prácticas sostenibles y productivas sobre el mismo, ya que en promedio, la generación de este residuo corresponde al $45 \%$ del total del fruto fresco, el cual presenta características favorables, para el crecimiento del hongo comercial Pleurotus ostreatus.

Finalmente, se puede concluir que, la muestra deslignificada mediante el pre-tratamiento biológico presentó mayor concentración de azúcares con un 89,51 $\mathrm{mg} \mathrm{L}^{-1}$ inicialmente, y con mayor transformación de los mismos después del proceso de fermentación alcohólica $72,46 \mathrm{mg} \mathrm{L}^{-1}$.

\section{AGRADECIMIENTOS}

A nuestra alma mater Universidad Mariana, y en especial, al Programa de Ingeniería Ambiental por ser nuestro instrumento de formación ética y académica.

A la Fundación Social Pasto, Dr. Guillermo Torres Daza y a su equipo de trabajo por su apoyo técnico y financiero.

Al Centro Estudiantil de Investigaciones de la Universidad Mariana, Convocatoria San Francisco de Asís 2016.

Agradecemos al Semillero de Investigación TARIPAHUASI, a la M.Sc. Jenny Lucia Huertas Delgado y a la M.Sc. María Patricia Obando Enríquez, por su participación en los procesos investigativos y aporte académico.

Al Director de la Unidad de Proyección Social de la Universidad Mariana, M.Sc. Arturo Obando Ibarra por la difusión del proyecto.

\section{REFERENCIAS}

Ballesteros, I., Negro, M.J., Oliva, J.M., Cabañas, A., Manzanares, P., Ballesteros, M., 2006. Ethanol production from steam-explosion pretreated wheat straw. Applied Biochemistry and Biotechnology 129-132, 496-508.

Balseca, D., Cabrera, J.C., 2011. Producción de biogás a partir de aguas mieles y pulpa de café. Tesis de grado de Licenciado en Ingeniero en Desarrollo Socioeconómico y Ambiente, Escuela Agrícola Panamericana, Zamorano, Honduras. 20 p.

Bonifacino, S., 2012. Estudios de des-lignificación de bagazo 
de caña de azúcar para la producción de bioetanol. Tesis Lic. Bioquímica, Facultad de Ciencias, Universidad de la República, Montevideo. 41 p.

Cardona, L.F., Bedoya, A., 1996. Producción de orellanas (Pleurotus ostreatus), deshidratadas y condimentadas. Tesis de Magíster en Ciencia y Tecnología de Alimentos, Universidad Nacional de Colombia, Facultad de Ciencias Agropecuarias, Medellín, Colombia. 88 p.

Chávez-Sinfonte, M., Domine, M., 2013. Lignina, estructura y aplicaciones: métodos de despolimerización para la obtención de derivados aromáticos de interés industrial. Avances en Ciencias e Ingeniería 4(4), 15-46.

Davis, S., Dohleman, F., Long, S., 2010. The global potential for Agave as a biofuel feedstock. Global Change Biology Bioenergy 3(1), 68-78. https://doi.org/10.1111/ j.1757-1707.2010.01077.x

Alcaldía Municipal de Buesaco, 2008. Esquema de ordenamiento territorial Buesaco-Nariño 2008-2015. Buesaco. 343 p. http://cdim.esap.edu.co/BancoConocimiento/B/ buesaco_-_narino_-_eot_-_2008/buesaco_-_narino_-_ eot_-_2008.asp

Fajardo, E., Sarmiento, S., 2007. Evaluación de melaza de caña como sustrato para la producción de Saccharomyces cerevisiae. Tesis de grado de Microbiólogo Industrial, Pontificia Universidad Javeriana, Facultad de Ciencias, Bogotá, Colombia. 120 p.

Federación Nacional de Cafeteros de Colombia, 2013. Comportamiento de la Industria Cafetera Colombiana. Federación Nacional de Cafeteros de Colombia Chinchiná, Caldas, Colombia. 1-64 p. https://www.federaciondecafeteros.org/static/files/Informe_Industrial_2013_Web. pdf

Fernández, J., Gutiérrez, F., Del Río, P., San Miguel, G., Bahillo, A., Sánchez, J.M., Ballesteros, M., Vázquez, J., Rodríguez, L., Aracil, J., 2015. Tecnologías para el uso y transformación de biomasa energética. Editorial Mundi-Prensa, Madrid, España.

García Criado, B., León Morán, L., García Ciudad, A., 1977. Determinación directa de proteína, NDF, ADF, lignina, DNDF y DMD en plantas herbáceas mediante reluctancia de infrarrojos. Pastos 7(1), 112-126.

Gonzáles, J., Molina, M., 2009. Estudio de los factores que afectan la hidrólisis enzimática y el proceso fermentativo para la producción de alcohol a partir de la papa (So- lanum tuberosum). Revista de Ingeniería 16(1), 27-37.

Juri Awad, S., 2011. Sacarificación y fermentación simultánea para la producción de bioetanol de segunda generación, mediante pretratamientos alternativos: líquidos iónicos reciclados y hongos de pudrición blanca. Tesis de grado de Ingeniero Civil en Biotecnología, Universidad de Chile. 66 p.

Marqués, G., Guiraum, P., Alfonso, G., 1999. Deslignificación de pasta kraft de eucalipto con el polioxometalato $\mathrm{SiW}_{11} \mathrm{M}$ $\mathrm{n}^{\mathrm{III}}$ en un proceso multietapas con peroxidasa versátil de Pleurotus eryngii. Tesis de Máster en Química Industrial y Medioambiental, Universidad de Sevilla, Sevilla, España. $37 \mathrm{p}$.

Núñez, R., 2015. Acción degradativa de hongos basidiomicetes sobre residuos agroindustriales de cultivos de café y plátano. Lic. Esp. Microbiología Industrial, Universidad Católica de Manizales, Caldas, Colombia. 126 p.

Ospina, A., Palacios, M., 1994. Efecto del cultivo de levaduras sobre la carga orgánica de los efluentes de Sucromiles S.A. Tesis de grado de Ingeniería en Microbiología, Universidad del Valle, Cali, Colombia. $172 \mathrm{p}$.

Rodríguez, N., Jaramillo, C., 2004. Cultivo de hongos comestibles del género Pleurotus sobre residuos agrícolas de la zona cafetera. Centro Nacional de Investigaciones de Café (CENICAFE), Chinchiná, Caldas, Colombia. pp. 1-61. http://biblioteca.cenicafe.org/bitstream/10778/582/1/027.pdf

Rodríguez, N., 2007. Balance energético en la producción de etanol a partir de la pulpa y el mucílago de café y poder calorífico de los subproductos del proceso del cultivo de café. Centro Nacional de Investigaciones de Café (CENICAFE), Chinchiná, Caldas, Colombia, pp. 1-14.

Rodríguez, N., Zambrano, D., 2010. Los subproductos del café: Fuente de energía renovable. Centro Nacional de Investigaciones de Café (CENICAFE), Chinchiná, Caldas, Colombia, pp. 1-18.

http://biblioteca.cenicafe.org/bitstream/10778/351/1/ avt0393.pdf

Sánchez, O., Cardona, C., 2005. Producción biotecnológica de alcohol carburante II: integración de procesos. Interciencia 30(11), 679-687.

Salcedo J., Galán, J., Pardo, L., 2012. Hidrólisis enzimática de residuos de la cosecha de caña de azúcar. Revista Colombiana de Biotecnología 14(1), 171-181. 